\title{
Measuring the effect of multiple eye fixations on memory for visual attributes
}

\author{
JOHN PALMER and CYNTHIA T. AMES \\ University of Washington, Seattle, Washington
}

\begin{abstract}
Because of limited peripheral vision, many visual tasks depend on multiple eye fixations. Good performance in such tasks demonstrates that some memory must survive from one fixation to the next. One factor that must influence performance is the degree to which multiple eye fixa. tions interfere with the critical memories. In the present study, the amount of interference was measured by comparing visual discriminations based on multiple fixations to visual discriminations based on a single fixation. The procedure resembled partial report, but used a discrimination measure. In the prototype study, two lines were presented, followed by a single line and a cue. The cue pointed toward one of the positions of the first two lines. Observers were required to judge if the single line in the second display was longer or shorter than the cued line of the first display. These judgments were used to estimate a length threshold. The critical manipulation was to instruct observers either to maintain fixation between the lines of the first display or to fixate each line in sequence. The results showed an advantage for multiple fixations despite the intervening eye movements. In fact, thresholds for the multiple-fixation condition were nearly as good as those in a control condition where the lines were foveally viewed without eye movements. Thus, eye movements had little or no interfering effect in this task. Additional studies generalized the procedure and the stimuli. In conclusion, information about a variety of size and shape attributes was remembered with essentially no interference across eye fixations.
\end{abstract}

Many visual tasks require multiple eye fixations. To introduce the significance of multiple fixations, consider three observations: (1) vision declines with eccentricity from the center of fixation, (2) the eyes move in fixation sequences with intervening saccades, and (3) when the eyes move, widely separated objects can be perceived and remembered. These observations lead to the hypothesis that individual fixations provide samples of the visual world that are remembered and combined to support tasks such as reading, search, and recognition. More specifically, the proximal stimulus for perception is an individual fixation. Some information from the fixation is maintained in memory across eye movements. This information is combined with information from later fixations in the final percept. For lack of an established name, this commonly held hypothesis is referred to as the fixationsampling hypothesis.

In this article, we consider only the memory component of this hypothesis. Furthermore, only one aspect of memory is addressed: To what extent do multiple fixations interfere with memory from a particular fixation?

\section{Previous Research}

The effect of multiple fixations on perception and memory has been addressed by a diverse range of studies.

We thank James Cutting, David Irwin, Colin MacLeod, Sandy Pollatsek, and several reviewers for useful comments on earlier versions of this article. We also thank David Irwin and Misha Pavel for many helpful suggestions during the course of the research. Please send correspondence to J. Palmer, Department of Psychology, NI-25, University of Washington, Seattle, WA 98195 (e-mail: jpalmer@u.washington.edu).
Some have been concerned with the integration of information from eye fixation to eye fixation. Others have been concerned with more narrowly defined phenomena such as direction constancy from eye fixation to eye fixation. Here we briefly review a few of these studies with our specific question in mind: How much, if at all, do multiple fixations interfere with memory?

Integration of information across saccades. In integration paradigms, a pair of complex displays is presented sequentially and a subject must integrate the two displays to perform a task. For example, Di Lollo (1977) studied the temporal properties of visual memory using a task where a matrix of dots was presented in two displays such that, when the displays were integrated, only a single dot was missing. Without eye movements, observers reported the location of the missing dot with considerable accuracy when the interval between displays was limited to tens of milliseconds. Irwin and his colleagues (Irwin, Yantis, \& Jonides, 1983; see also O'Regan \& Lévy-Schoen, 1983; Rayner \& Pollatsek, 1983) used this paradigm to determine if such a visual memory persisted when subjects had to move their eyes between the presentations of the two displays. The answer was no. Similar results have been obtained with priming and masking paradigms that resemble integration (Irwin \& Brown, 1988; Sun \& Irwin, 1987). Thus, when complex patterns were viewed, eye movements interfered with visual memory and/or integration.

A variation on this paradigm addressed whether eye movements interfered specifically with visual memory. Instead of requiring subjects to integrate two displays, Ir- 
win (1991; Irwin, Zacks, \& Brown, 1990) required a same-different judgment of the two displays (cf. Phillips, 1974). When this was done across eye movements, performance was above chance, indicating that some memory survived. But, performance was much less than that observed without eye movements (Irwin, 1991; Irwin et al., 1990). Thus, this paradigm showed that eye movements interfered with memory.

Visual direction constancy. Visual direction constancy is the ability to judge the position of objects accurately despite changes in eye position. This task has been studied largely as a constancy but also has implications for visual memory. For example, Matin (1986; Matin, Matin, \& Pearce, 1969; Matin, Matin, \& Pola, 1970; see also Hansen \& Skavenski, 1985; O'Regan, 1984) presented a flash of light in an otherwise dark room, required the observer to move his/her eyes, and then presented another flash of light. The observer's task was to judge the position of the second light relative to the first. Matin showed that performance was inaccurate when the flashes were within a second of the eye movement. For longer intervals, performance was not affected by the eye movement. Thus, memory for position must not have been affected by the eye movement.

Other paradigms. Several other paradigms are relevant. One is to measure the effects of performing a task with and without eye movements. For example, Kowler and Steinman $(1977,1979)$ had observers count objects while maintaining fixation or moving their eyes freely. Counting was more accurate when the eyes were free to move unless the displays were very small. Eye movements have also been shown to facilitate shape-recognition tasks where the shapes are several degrees in extent (Schlingensiepen, Campbell, Legge, \& Walker, 1986). Another interesting approach to showing successful form perception across multiple fixations can be found in Hayhoe, Lachter, and Feldman (1991). The improved performance with eye movements provides indirect evidence that some kind of memory survives across fixations.

In another paradigm, subjects performed a task with or without a previous view of an object in peripheral vision. McConkie and Rayner (1975; see also McConkie, Zola, Blanchard, \& Wolverton, 1982; Rayner, 1975) used an eye-position-dependent display to limit the peripheral vision of readers to only the letters of text around their current fixation. Limited peripheral vision affected reading behavior only when the visible area was restricted to less than two or three words' worth of characters. There was no sign of memory for words farther in the periphery. On the other hand, the studies did show an advantage of previewing one word to the right of fixation. On the basis of these studies, the authors argued that the remembered information was a nonvisual code for the beginning letters of the peripherally viewed word (Balota, Pollatsek, \& Rayner, 1985; McConkie \& Zola, 1979; Rayner, McConkie, \& Zola, 1980). This paradigm has also been used to study object recognition and visual search (Henderson, Pollatsek, \& Rayner, 1987; Pollat- sek, Rayner, \& Collins, 1984; Rayner \& Fisher, 1987). The effects of a peripheral preview provide further indirect evidence that some kind of memory persists from previous fixations.

Summary. The studies of visual direction constancy provide an example where there is essentially no interference from eye movements. The studies of integration paradigms provide an example where there is essentially complete interference for integrated complex patterns. Other paradigms provide indirect evidence for some memory surviving interference from eye movements. However, none of these paradigms provides a systematic way to measure the degree of interference due to the eye movements.

\section{A Study-Test Paradigm}

The goal of the present work was to measure how much eye movements interfere with memory for a variety of visual attributes. The experiments differed from previous work in quantifying the degree of interference. The experiments also differed from previous work in systematically addressing a variety of stimuli and in trying to isolate memory limitations from other performance limitations. These goals motivated the study-test paradigm illustrated in Figure 1. A display was presented for a second or so with two widely separated objects such as the vertical lines shown. The observer was instructed to fixate each line in turn and to remember a particular attribute of each. Then the observer returned his/her gaze to a central fixation, and a second display was shown with a comparison stimulus for one of the lines studied. The illustrated task was to compare the test line with the study line on the right. Is the test line longer or shorter? To perform well on this task, the observer must remember

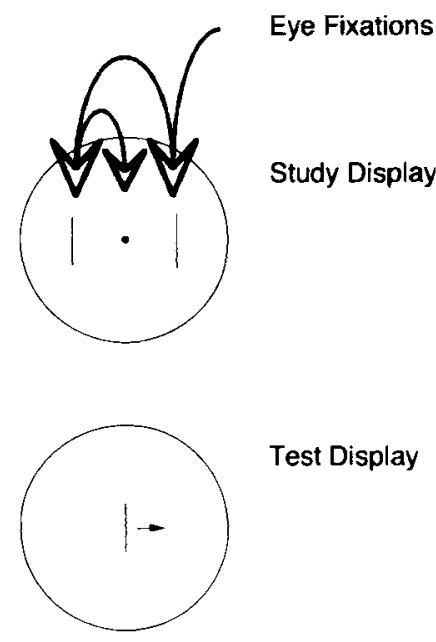

Figure 1. Schematic illustration of the task used in these experiments. The observers scanned a set of stimuli in a study display and then compared a test stimulus with the study stimulus, indicated by an arrow. In the prototype length task, the observers judged if the single test line was longer or shorter than the indicated study line. 


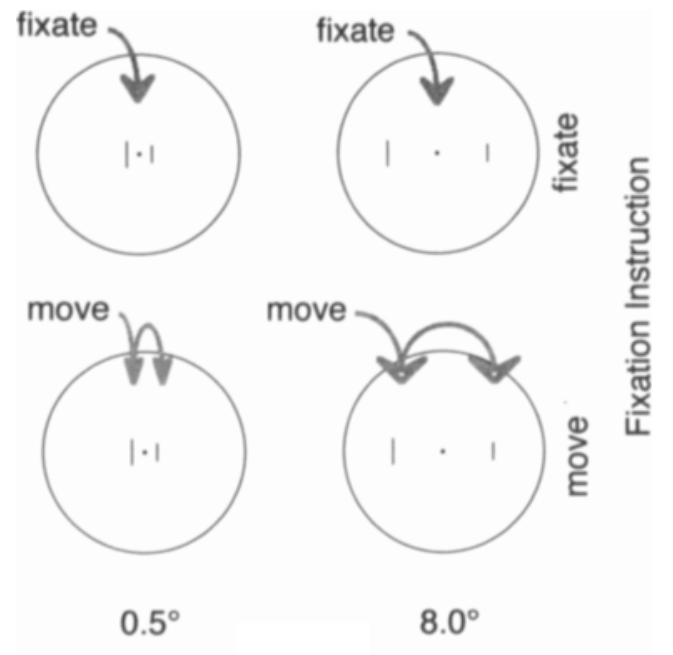

\section{Eccentricity}

Figure 2. Schematic illustration of the four conditions in each experiment. The eccentricity of the stimuli was factorially combined with instructions to either fixate the center of the display or to move one's gaze to each stimulus in turn.

information from the fixation of each line. A similar study-test paradigm without multiple eye fixations was described in Palmer (1990).

To evaluate the amount of interference from multiple fixations, we defined the four conditions illustrated in Figure 2. Instructions and stimulus eccentricity were factorially manipulated. Instructions in the movement condition were to move the eyes from stimulus to stimulus and back to the central fixation point; instructions in the fixation condition were to maintain central fixation throughout the trial. Eccentricity was manipulated by placing the stimuli either $8.0^{\circ}$ or $0.5^{\circ}$ from the central fixation point. Of these four conditions, the critical condition has the movement instructions and the large eccentricity. The other three conditions provide two controls.

The first control provides a baseline for the performance expected if no memory survives an eye movement. Consider the two large-eccentricity conditions. The condition with fixation instructions limits observers to viewing the stimuli with peripheral vision. This peripheral view is the same as the last view in the movement condition because the movement condition always ended with the observer returning to central fixation. Thus, even if eye movements interfere completely, performance in the movement condition should not be worse than in the fixation condition. In summary, the fixation condition provided a lower bound on the performance expected in the movement condition.

The second control provides a baseline for the performance expected if memory from previous fixations is perfect. Consider the small-eccentricity conditions illustrated on the left side of Figure 2. Here the eccentricity of the stimuli approaches foveal viewing. Foveal viewing sets an upper bound on the performance expected in the movement condition. If eye movements did not interfere with memory, then performance in the movement condition should be independent of the eccentricity of the stimuli from the central fixation point. This is because they are all viewed foveally one after the other. Performance for a foveal view without eye movements can be estimated by examining the effects of eccentricity in the fixation conditions. Such eccentricity effects have been studied for many tasks and found to be essentially linear (e.g., Cowey \& Rolls, 1974; Farrell \& Desmarais, 1990; Klein \& Levi, 1987). Hence, for the fixation conditions, one can linearly extrapolate the threshold for a $0^{\circ}$ eccentricity from the $0.5^{\circ}$ and $8.0^{\circ}$ conditions and compare it to the movement condition. If there is no interference, then both movement conditions should equal the extrapolated foveal thresholds.

The two extreme predictions just described are illustrated by the graphs in Figure 3 . In both graphs the lengthdifference threshold is plotted as a function of eccentricity. The dotted line is for the fixation conditions where observers are to maintain a steady central fixation, and the solid line is for the movement conditions where observers are to move their gaze to foveate each stimulus individually. An effect of eccentricity is expected in the fixation conditions. If there is no interference, then the results of the top graph are predicted. The eccentricity

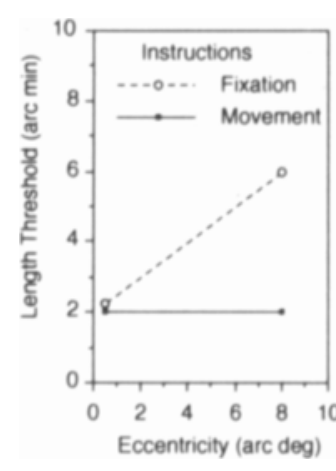

\section{No \\ Interference}

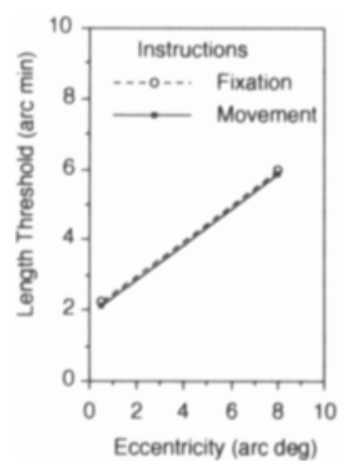

Complete

Interference

Figure 3. Illustration of two possible outcomes of these experiments. Each outcome is illustrated by a graph of the length threshold as a function of eccentricity with the instruction to fixate or move as the curve parameter. If memory is perfect, then the effect of eccentricity will be eliminated in the movement condition; if there is no memory, then the effect of eccentricity will be the same in the movement and fixation conditions. 
effect will be eliminated for the movement condition, and the thresholds will match those extrapolated from the fixation conditions for zero eccentricity. In contrast, if there is complete interference, then the results of the bottom graph are predicted. Because the last view of the study stimulus is identical in the two cases, the thresholds should show identical eccentricity effects. The quality of any memory can be measured relative to these extreme predictions. In particular, interference can be measured by the degree to which movement instructions reduce the effect of eccentricity. Measuring the degree of interference is the goal of this paper.

What might be expected in this paradigm on the basis of previous results? Generalizing from integration paradigms suggests complete interference, generalizing from visual direction paradigms suggests no interference, and generalizing from the same-different experiments of Irwin (1991) suggests some interference. The next section introduces the details of the methodology. The following set of experiments introduces the paradigm with the two line stimuli and then generalizes it to other procedures and stimuli.

\section{General Method}

Observers. Observers for each experiment were selected from 7 experienced, 20-40-year-old observers. Most were graduate students, each was paid $\$ 5$ per hour, and all had normal or correctedto-normal visual acuity. The observers were identified by a set of noncontiguous numbers that were the same as those used in related studies (Palmer, 1988, 1989, 1990, 1991; Palmer, Ames, \& Lindsey, in press).

Apparatus. The displays were controlled by a Hewlett-Packard Model 9817 computer and were presented on a 14-in. cathode-ray tube (Hewlett-Packard Model 35122A). The tube has a P31 phosphor, a $60-\mathrm{Hz}$ raster refresh, and a $512 \times 390$ pixel resolution. This tube was optically combined with a $22^{\circ}$-diam, low-luminance disk to produce a composite display of a few computer-generated stimuli on the background of a relatively large disk. The disk had a luminance of $5 \mathrm{~cd} / \mathrm{m}^{2}$, which precluded any view of the unilluminated frame of the tube. The displays on the tube combined with this field to produce stimuli with a combined luminance of $200 \mathrm{~cd} / \mathrm{m}^{2}$. Outside of the low-luminance disk was a dark $\left(<0.03 \mathrm{~cd} / \mathrm{m}^{2}\right)$ surround that filled the remaining visual field. The tube and disk were $78 \mathrm{~cm}$ in front of the observer.

Stimuli. In the prototype experiment, the stimuli were vertical lines. As shown in Figure 4, these stimuli appeared in both a study display and a test display. In the test display, only a single line appeared, and its length was always a standard $60^{\prime}$ of arc. In the study display, two lines were arranged at a fixed eccentricity on either side of a central fixation point. One of the two study lines served as a comparison, and the other was a distractor. The observer, of course, did not know which was which. The length of the comparison line varied in steps of $2^{\prime}$ of arc over a range of plus or minus approximately two times the estimated difference threshold. Taking Experiment 1 as an example, in all but the eccentric-fixation condition, the comparison lines were $54^{\prime}, 56^{\prime}, 58^{\prime}, 62^{\prime}, 64^{\prime}$, and $66^{\prime}$ of arc. In the eccentric-fixation conditions, the comparison stimuli were $48^{\prime}, 52^{\prime}, 56^{\prime}, 64^{\prime}, 68^{\prime}$, and $72^{\prime}$ of arc. The length of the distractor line was chosen from the same set of values as the comparison stimuli.

Procedure. The stimulus sequence is illustrated in Figure 4. The series of displays is shown with the instructed position of the eye illustrated with a large halftone arrow. There were three displays separated by blank intervals. The first display instructed the ob-

\section{Display Sequence}

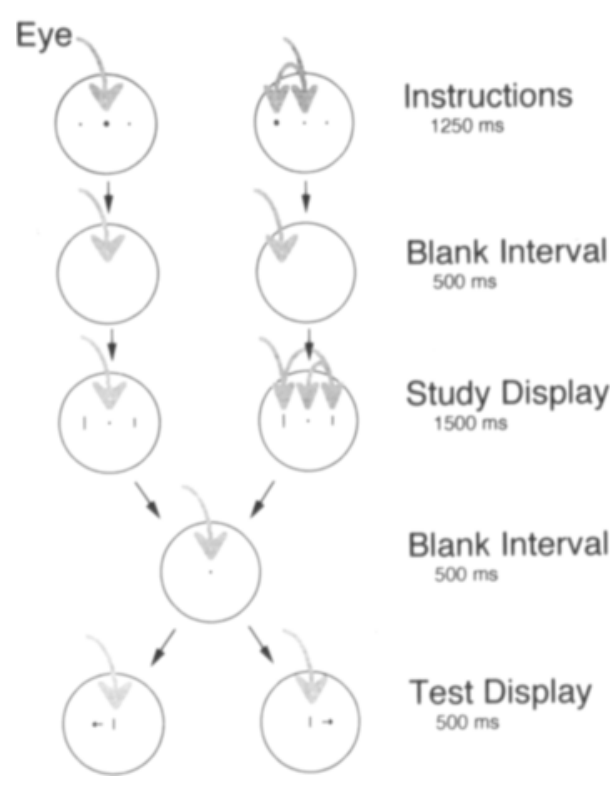

Figure 4. Schematic illustration of the sequence of displays used in the first experiment. An example of the fixation condition is shown on the left, and an example of the movement condition is showh on the right. The intended position of the observer's gaze is indicated by the halftone arrows.

server how to move his/her gaze by the size of the points in the fixation pattern. A larger point in the center indicated that fixation was to be maintained at the center throughout the trial; a larger point to the left indicated that fixation was to start with the left stimulus, then move to the right, and lastly return to center; a larger point to the right indicated that fixation was to start at the right stimulus, then move to the left, and lastly return to center. In short, the larger point marked the first fixation of the sequence. The first of these changes in gaze was to be made immediately, while the instructions were still displayed.

The study display followed an intervening blank display. It contained two lines of variable length, and the observer's task was to remember the lengths of these lines. If a trial was in a movement condition, then the observer made eye movements at this time. Otherwise, fixation was maintained at the center.

The test display followed another intervening blank display. It contained a single line and an arrow cue. The observer's task was to compare the single test line to the study line indicated by the arrow cue. For example, if the arrow pointed to the left, then the observer was to judge if the test line was longer or shorter than the line on the left side of the study display.

The timing of these displays is shown in Figure 4: 1,250 msec for the instructions, $500 \mathrm{msec}$ for the blank interval, $1,500 \mathrm{msec}$ for the study, $500 \mathrm{msec}$ for the next blank interval, and $500 \mathrm{msec}$ for the test. These durations gave the observer plenty of time to interpret the instructions and move his/her eyes. In pilot work, much shorter durations were used with similar results.

The observer made the line-length discrimination by pressing one key if he/she thought the test line was shorter than the indicated study line and another key if longer. There was no time pressure, and response-accuracy feedback was provided by tones. A psychometric function was formed by calculating the proportion of "'longer" responses as a function of the comparison length. The observed function was fit to a cumulative normal, and the difference threshold was defined as half the difference between the stim- 
ulus that produced 25\% "longer" responses and the stimulus that produced $75 \%$ "longer" responses. In addition, the point of subjective equality was defined as the stimulus that resulted in $50 \%$ "longer" responses. Specific comparison stimuli were chosen randomly from trial to trial according to an adaptive method of constant stimuli. More details of stimulus selection, analysis, and typical psychometric functions can be found in Palmer (1990). Trials were presented in blocks of 32 trials, and a day's session consisted of 12 blocks. Each observer participated in at least 10 sessions of general practice before providing any of the reported data. In addition, before each experiment, each observer participated in one or more sessions of specific practice in that experiment. This resulted in at least 3,300 trials of practice per observer.

\section{EXPERIMENT 1}

The first experiment followed the method described above. It provided a first measurement of the possible interference of multiple eye fixations.

\section{Method}

The general method was followed in all respects. The main conditions were the eccentricity of the stimuli and the instruction to move or not to move one's gaze. The eccentricities of the vertical lines were $0.5^{\circ}$ and $8.0^{\circ}$. Four sessions of data were collected in the four conditions, yielding 384 trials per condition per observer. Seven observers participated in the experiment, but 1 was dropped because she did not follow the eye-movement instructions as described below.

\section{Results}

Main results. Figure 5 shows the mean of 6 observers' length-difference thresholds as a function of eccentricity.

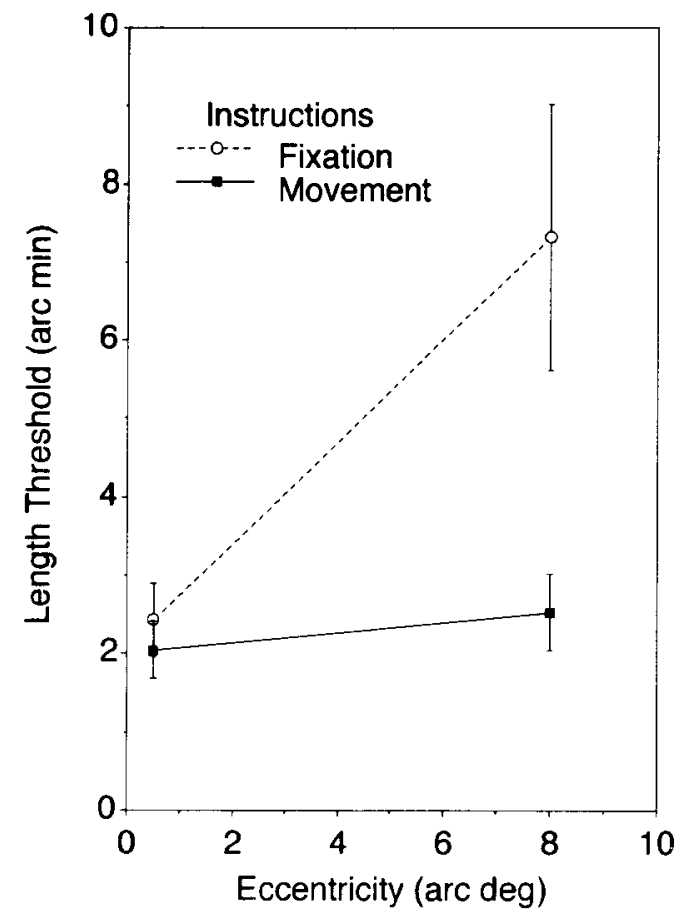

Figure 5. Results of Experiment 1. The mean length threshold as a function of eccentricity and instruction is shown. Each error bar indicates the standard error of the mean across observers.
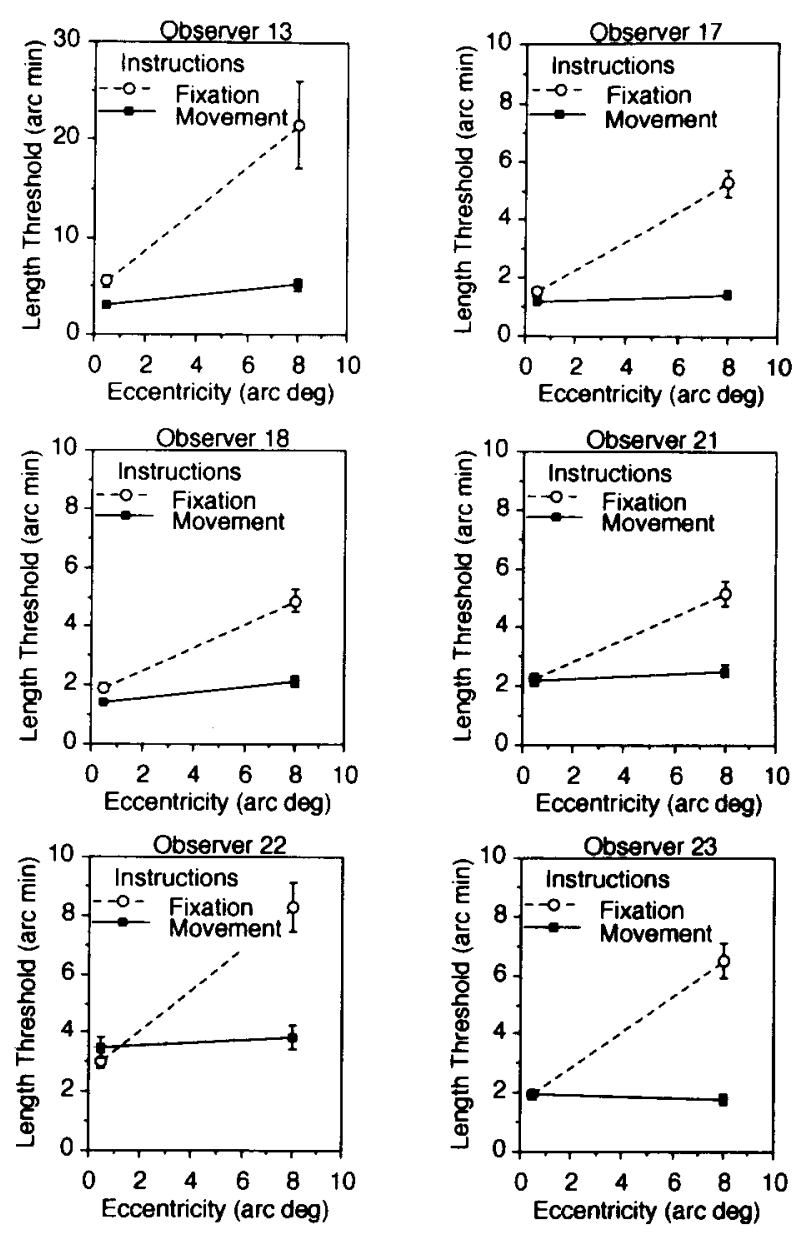

Figure 6. Results of Experiment 1. For each observer, an individual graph shows the length threshold as a function of eccentricity and instruction. Error bars for individual observer graphs are standard errors estimated by probit analysis. A larger scale ordinate is used for Observer 13.

The dotted line shows the thresholds in the fixation condition and replicates the expected effects of eccentricity. The solid line shows the thresholds in the movement condition. There was little or no effect of eccentricity when an observer moved his/her gaze. One way this interaction can be described is by the percentage that the movement instruction reduced the eccentricity effect. For example, in this experiment, the eccentricity effect can be summarized by a linear function with a slope of $0.013 \pm 0.005$ in the fixation condition and a slope of $0.001 \pm 0.001$ in the movement condition. The movement condition eliminated $91 \% \pm 4 \%$ of the effect found in the fixation condition. Clearly, eye movement improved performance more than it interfered with memory for previous fixations. These results were replicated in all of the individual observers, as shown in Figure 6. For each observer, the movement instructions reliably reduced the effect of eccentricity. From observer to observer, the percentage reduction ranged from $76 \%$ to $104 \%$.

There is another way to measure the effect of intervening eye movements. On each movement-condition trial, 
an observer gazed at one and then another study stimulus before returning to gaze at the center of the display where the comparison stimulus appears. Thus, depending on which study stimulus was tested, there were one or two intervening eye fixations. Did this fixation lag affect performance? The answer was no. For the 6 observers in Experiment 1, the difference between Fixation Lags 1 and 2 was $-0.2^{\prime} \pm 0.2^{\prime}$ of arc in the small-eccentricity condition and $0.0^{\prime} \pm 0.2^{\prime}$ of arc in the large-eccentricity condition. Furthermore, fixation lag did not have consistent effects in any of the other experiments reported in this article. This is not surprising once one accepts the lack of interference in the comparison between fixation and movement conditions. It provides a second independent piece of evidence that eye movements do not interfere.

Eye-movement measurements. These experiments manipulated instructions to fixate or move one's eyes. To interpret the results, it is critical that the observers followed these instructions. Consider the possibilities. If they ignored the instructions, then the instruction manipulation should have had no effect on performance. That possibility can be clearly rejected for the 6 reported observers. Each shows a large and reliable effect of instructions. Another possibility is that they usually followed instructions but could not always maintain fixation when instructed. This possibility can be rejected because each observer showed the expected large effect of eccentricity in the fixation condition. A final possibility is that the observers could fixate but failed to move their eyes correctly in the movement condition. This possibility seems unlikely given the result of essentially no eccentricity effect in the movement condition. The only way to have overcome the eccentricity effect is to have correctly moved one's eyes.

To further support these arguments, we directly observed eye movements during trials of the experiment. One or the other of the authors served as a judge and decided if an observer made an eye movement during a trial. The judge was blind to the condition in that trial. Using this procedure, a judge watched Observer 17 through a session of 384 trials. Observer 17 followed instructions on at least $98 \%$ of the large-eccentricity trials and replicated her previous threshold results with a $99 \%$ reduction in the eccentricity effect. For the other observers, we made measurements on a short session of 64 trials. With the exception of 1 observer, each observer followed instructions on almost every large-eccentricity trial. Of a total of 192 trials for the 6 observers, there were no cases of an eye movement during a fixation trial and only five cases of no eye movement being seen during a movement trial. In summary, the observers followed instructions in at least $96 \% \pm 1 \%$ of the large-eccentricity trials. For the small-ccentricity trials, these eye-movement measurements were less accurate, but still showed that the observers followed instructions on at least $86 \% \pm 2 \%$ of the trials. We suspect that the remaining $14 \%$ were largely trials in which the observers still followed instructions, but our eye-movement measurement was inaccurate.
The exception to this pattern was Observer 15, who appeared to make eye movements unpredictably on both movement and fixation trials. This observer also showed distinctly different threshold data, with little effect of instruction and reduced eccentricity effects in both instruction conditions. Thus, this observer was clearly not following instructions, and her data were excluded from further analysis. We conclude from both the threshold data and the eye-movement measurements that the other 6 observers did follow the eye-movement instructions.

\section{Discussion}

This experiment provided a first measurement of the amount of interference from multiple eye fixations on memory for a simple visual attribute. There was little or no such interference. This conclusion is based on performance with eye movements nearly matching foveal performance, the average of a $94 \%$ reduction in eccentricity effects, and the lack of a fixation lag effect. Any residual memory interference effect is small relative to the eccentricity effect that limits perception. For the conditions of this experiment, the advantage of avoiding eccentricity effects is overwhelmingly more important than any cost of memory interference.

\section{EXPERIMENT 2}

One feature of the first experiment was that the test line was always the standard length of $60^{\prime}$ of arc. This procedure guarantees that the test provided no information and that the comparison had to be based on the study lines. The complication is that this procedure allowed the observers to adapt a categorization strategy where they judged the study lines against a memory of the standard and only remembered if the study lines were longer or shorter than the standard. Many, but not all, observers reported using this strategy (see Palmer, 1990). For current purposes, we wished to show that the results were not specific to using this strategy. The procedure was modified so that there were nine standard line lengths that might be tested on a trial. This introduced uncertainty in the test standard, and the observers reported not being able to use the categorization strategy.

\section{Method}

The nine test standards varied from $44^{\prime}$ to $76^{\prime}$ of arc in $4^{\prime}$ intervals. Observers 17 and 21 participated in 12 sessions, yielding 132 trials per condition per observer. The standards were grouped into three ranges (small, $44^{\prime}, 48^{\prime}$, and $52^{\prime}$; middle, $56^{\prime}, 60^{\prime}$, and $64^{\prime}$; and large, $68^{\prime}, 72^{\prime}$, and $76^{\prime}$ ) to obtain 396 trials per grouped condition.

\section{Results}

Figure 7 shows separate graphs for Observers 17 and 21 and for the the test standards grouped into the three ranges of small, middle, and large. In all cases, there are eccentricity effects, and there are large reductions in the eccentricity effects with the movement instructions. The reductions of the eccentricity effect were reliable for both 

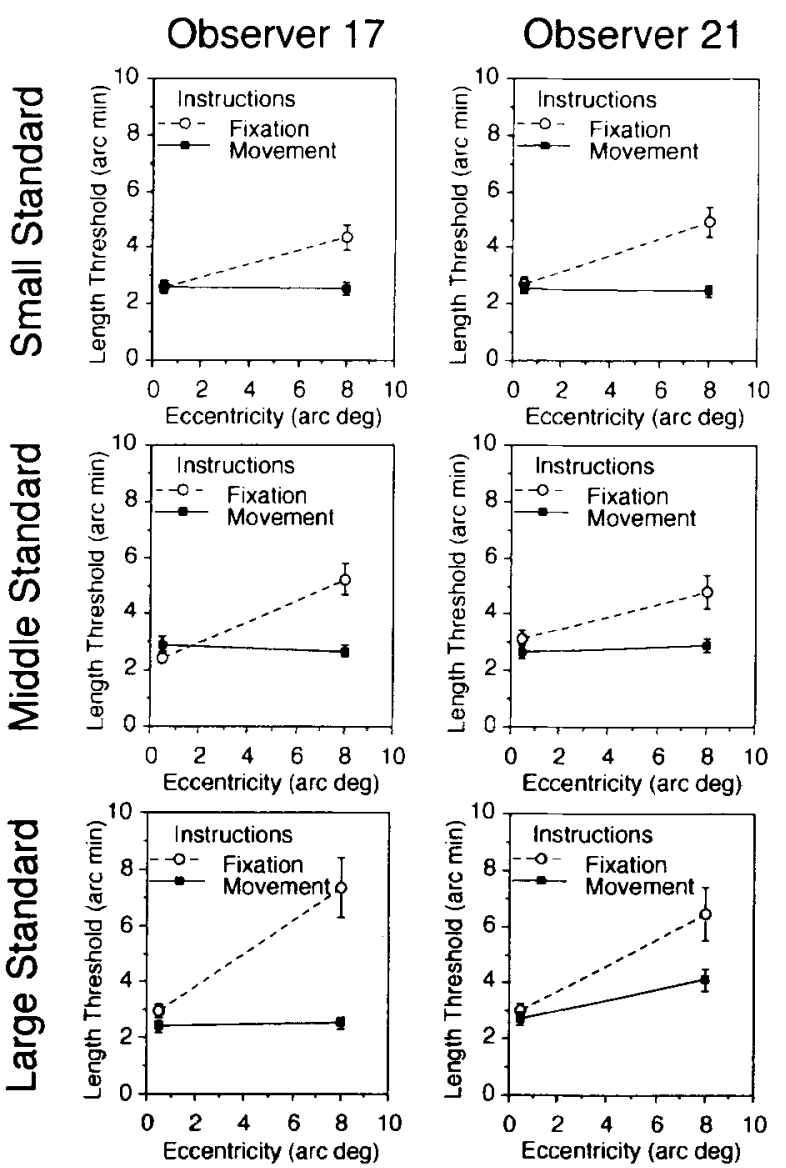

Figure 7. Results of Experiment 2. Graphs show replications of Experiment 1 for three different sets of test standards.

observers in all conditions. For example, for the middle standard, which was closest to Experiment 1, the reduction in the eccentricity effect was $107 \% \pm 4 \%$ and $104 \% \pm$ $26 \%$ for Observers 17 and 21 , respectively. ${ }^{1}$ These values are essentially identical to the $98 \% \pm 4 \%$ and $92 \% \pm 13 \%$ reductions found for these observers in Experiment 1.

\section{Discussion}

Experiment 2 replicates the effects of Experiment 1. A large amount of test uncertainty did not change the results. There is still little or no interference with the memory for these line lengths. Categorization is not necessary to avoid interference from intervening eye movements.

\section{EXPERIMENT 3}

In Experiment 3, we extended Experiment 1 by displaying four stimuli and measuring performance with up to four fixations. It seemed possible that additional stimuli or additional fixations might introduce an interference effect even if one does not occur for two stimuli and two fixations.

\section{Method}

Figure 8 illustrates the four vertical lines used as stimuli. They were presented at eccentricities of $1^{\circ}$ or $5^{\circ}$ and centered on an imaginary circle at clock positions of $1,4,7$, and 10 o'clock. As before, the instructions were either to maintain fixation at the center of the display or to move one's gaze from line to line. Specifically, the instructions indicated the stimulus location to fixate initially. When the study display appeared, the observer was to gaze at each stimulus by moving his/her gaze in a clockwise direction and return to the central fixation point before the display ended. Because of the four study lines, the study duration was extended to $3 \mathrm{sec}$. Otherwise, the same procedures were followed as in Experiment 1. Two observers participated in eight sessions, yielding 512 trials per condition per observer.

\section{Results}

Figure 9 shows graphs for Observers 18 and 22. Each graph replicates the expected eccentricity effect and shows it to be essentially eliminated with the movement instruction. The movement instructions reduced the eccentricity effect by $94 \% \pm 15 \%$ and $136 \% \pm 26 \%$ for Observers

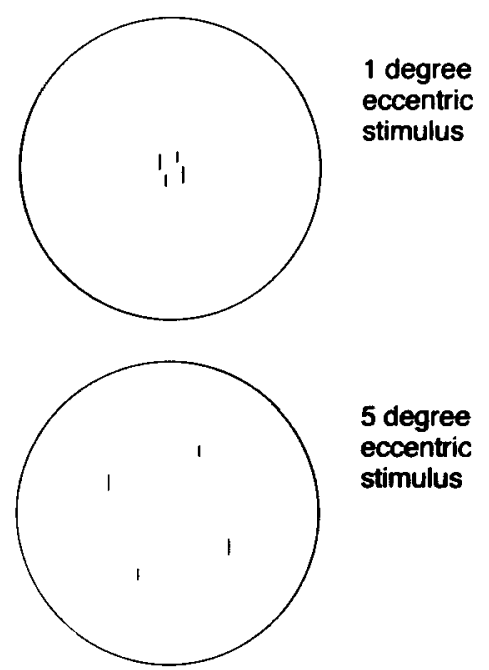

Figure 8. Scale drawing of the displays used in Experiment 3. Both $1^{\circ}$ and $5^{\circ}$ eccentricity conditions are shown.
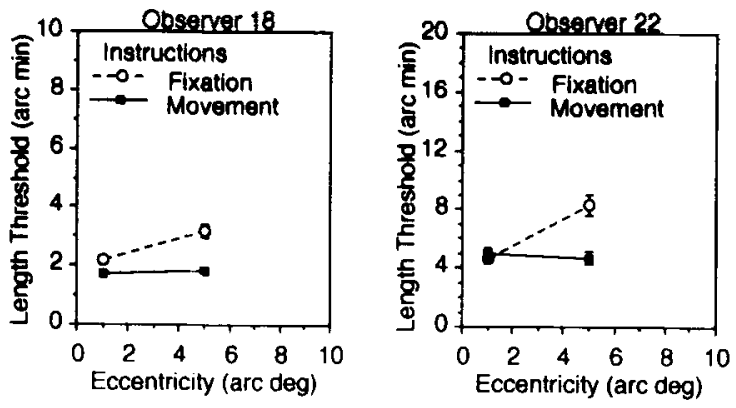

Figure 9. Results of Experiment 3. The graphs show length threshold as a function of eccentricity and instruction for Observers 18 and 22. This experiment used displays of four stimuli instead of the two stimuli used in Experiments 1 and 2. 
18 and 22 , respectively. ${ }^{2}$ There was also no systematic effect of fixation lag: for the movement-instruction conditions and both eccentricities, Fixation Lags 1-4 yielded respective thresholds of $1.75^{\prime}, 1.73^{\prime}, 1.66^{\prime}$, and $1.71^{\prime}$ of arc for Observer 18 and 3.7', 5.9', 5.9', and 4.9' of arc for Observer 22. Thus, increasing the number of stimuli to four and the number of intervening fixations to four did not change the results. A set of eye-movement measurements was made on a short session of 64 trials to confirm that the observers followed instructions in this new experiment. Both observers were seen to correctly follow instructions in all of the large-eccentricity trials. Thus, both the pattern of threshold data and the direct measurements indicate that the observers were able to follow instructions.

\section{Discussion}

This experiment generalized the results of Experiment 1 to four stimuli and four fixations. Even a sequence of four fixations does not significantly interfere with memory for the sizes of these lines. This is not to say that performance with four lines or four fixations is as good as that with fewer lines or fewer fixations. This experiment was not intended to measure capacity. Rather, this experiment measured the effect of eye movements with a constant set of stimuli in both the movement and fixation conditions. Thus, although there are capacity limits, they do not arise from the interference of multiple eye movements and fixations.

By comparing the results of Experiments 1 and 3, we can calculate a crude estimate of the effect of increasing the number of stimuli and fixations. Because the eccentricities were different, we extrapolated the results of each observer to an eccentricity of $0^{\circ}$. For the movementinstruction conditions, we found the following results. The estimated thresholds for two and four stimuli were, respectively, $1.38^{\prime}$ and $1.71^{\prime}$ of arc for Observer 18 and $3.23^{\prime}$ and $4.77^{\prime}$ of arc for Observer 22. This represents $24 \%$ and $48 \%$ increases in threshold for Observers 18 and 22 , respectively. Given the uncertainties of this comparison, these increases are consistent with the $41 \%$ increase (square root of 2) expected for a doubling of the number of stimuli on the basis of Palmer (1990). Thus, these results are consistent with similar capacity limits for brief displays and for multiple eye fixations.

\section{EXPERIMENT 4}

Experiment 4 was intended to replicate Experiment 1, but with a different judgment of a different stimulus. Three judgments were investigated: (1) a relative-orientation judgment, (2) a rectangle-height judgment that required a similar judgment to the line-length task but used outline rectangles as stimuli, and (3) a rectangle-shape judgment where observers judged the relative elongation of rectangles.

\section{Method}

The procedure followed Experiment 1 except for the substitution of three new tasks. In each case, 2 observers participated in four sessions, yielding 384 trials per condition per observer.

Relative orientation. The observer's task was to judge the relative orientation of two small squares. The standard stimulus is illustrated at the top of Figure 10. Two squares that subtended $6^{\circ}$ of arc were presented. In the standard, the squares were separated by $48^{\prime}$ of arc vertically and $30^{\prime}$ of arc horizontally. Measured another way, the two squares had a separation of $57^{\prime}$ of arc and a relative orientation to the vertical of $32^{\circ}$. Comparison stimuli varied in the horizontal separation of the squares by $2^{\prime}, 4^{\prime}$, or $6^{\prime}$ of arc, except for the large-eccentricity-fixation condition, where they varied by $4^{\prime}, 8^{\prime}$, or $12^{\prime}$ of arc. For comparison to other tasks, orientation thresholds were measured in terms of the horizontal shifts in position. For these stimuli, a shift in position of $1^{\prime}$ of arc was approximately a $0.4^{\circ}$ change in relative orientation. Eccentricity from the central fixation point to the inside edges of these stimuli was either $1.0^{\circ}$ or $7.73^{\circ}$, which yields an eccentricity to the centers of the stimuli of approximately $1.25^{\circ}$ or $8^{\circ}$.

Rectangle height. The observer's task was to judge the height of an outline rectangle. The standard test rectangle is illustrated in the middle of Figure 10 by the solid lines. It had a height of $60^{\prime}$ of arc and a width of $30^{\prime}$ of arc. The comparison stimuli varied only in height by $\pm 2^{\prime}, 4^{\prime}$, or $8^{\prime}$ of arc. The eccentricity of the center of the rectangles from the central fixation point was about $0.75^{\circ}$ and $8.0^{\circ}$. The exact eccentricity was defined by fixing the inside edge of the rectangles to be either $0.5^{\circ}$ or $7.83^{\circ}$. This experiment was nearly identical to Experiment 1 , except that the stimuli were outline rectangles instead of lines.

Rectangle shape. The standard test rectangle is illustrated at the bottom of Figure 10. It is the same as that in the rectangle-height condition. Comparison rectangles were constructed by elongating the rectangle either vertically or horizontally. A horizontal elongation is illustrated by the dotted lines. Both the width and the height were changed; the width was increased by a certain amount and the height was decreased by the same amount. This preserved the perimeter of the rectangle and almost preserved the area. Comparison stimuli had elongations in each dimension of $2^{\prime}, 4^{\prime}$, or $6^{\prime}$ of arc. Observers made a forced choice between a vertical and a horizontal elongation. The threshold was described in terms of the

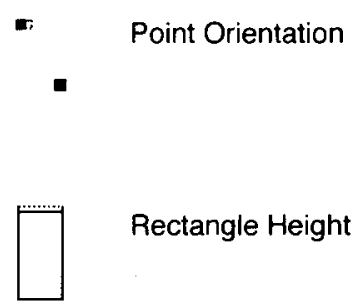

Rectangle Shape

Figure 10. Schematic illustration of the standard and comparison stimuli used in Experiment 4 . In the point-orientation task, the observers judged the relative orientation of two points. In the rectangle-height task, the observers judged the relative height of the rectangles. In the rectangle-shape tasks, the observers judged the relative elongation of the rectangles. 
change in the linear dimensions to make it easier to compare to those with line length. The eccentricity of the rectangles was the same as with rectangle-height judgments.

Performing the rectangle-shape task according to the methods of Experiment 1 revealed an artifact. Eccentricity was confounded with the exact location of the stimulus in the visual field. For example, the rectangles were distorted when displayed near the edge of the display device. To control for such effects, the positions of the stimuli were changed as illustrated at the top of Figure 12. In this display, one rectangle always appeared at the exact center of the display and the other rectangle appeared to one side or the other. The eccentricity from the center of the rectangles to the fixation point was $0.75^{\circ}$ and $4.08^{\circ}$. This method allowed for an analysis of just the rectangle that always appeared at the center of the display.

\section{Results}

The results of two judgments are shown for Observers 18 and 23 in Figure 11. In all cases there was an effect of eccentricity, and in all cases this effect was reduced in the movement-instruction condition. The reduction was $75 \% \pm 10 \%$ and $87 \%^{3}$ for relative orientation and $77 \% \pm 6 \%$ and $89 \% \pm 9 \%$ for rectangle height. These values are for Observers 18 and 23, respectively.

The results of the rectangle-shape judgment were different depending on the position of the rectangle and are illustrated in Figure 12. If the rectangle was in the center of the display, there was a substantial reduction of the eccentricity effect as found with other stimuli. The reduction was at least $75 \%^{4}$ for Observer 18 and $88 \%$ for Observer 23. In contrast, if the rectangle was to the side of the display, then the reduction was less, $28 \%$ for $\mathrm{Ob}$ server 18 and $73 \%$ for Observer 23 . In the latter case,

\section{Observer 18}
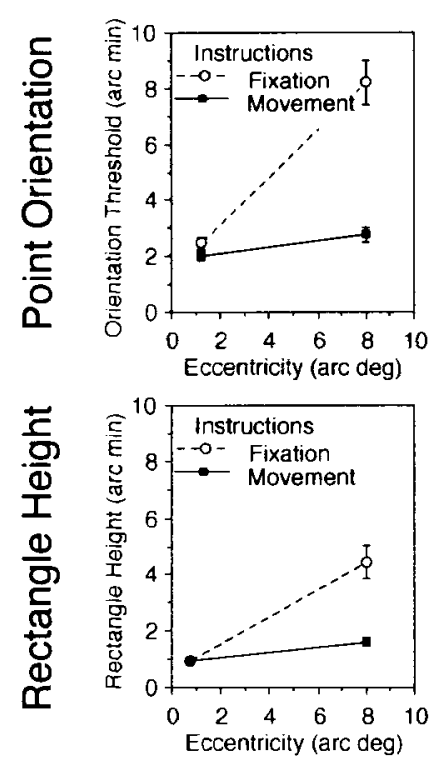

\section{Observer 23}
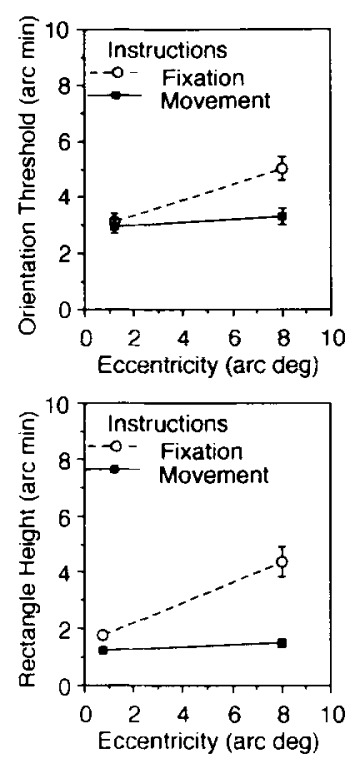

Figure 11. Results of Experiment 4. Graphs show replications of Experiment 1 for two new tasks. To facilitate comparison to other experiments, the threshold for each task is measured by the magnitude of a one-dimensional change in the comparison stimuli (see text).

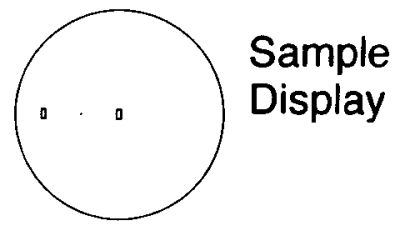

Observer 18

Observer 23
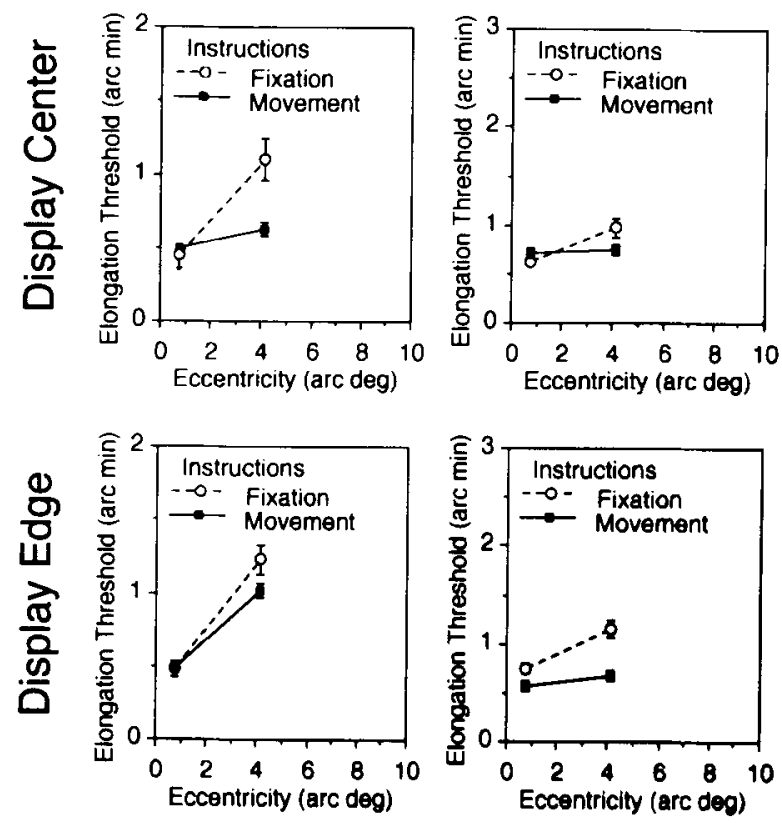

Figure 12. Results of Experiment 4. At the top of the figure is a schematic illustration of the display used in the rectangle-shope task. At the bottom of the figure are graphs of the elongation threshold as a function of eccentricity and instruction for 2 observers. The top two graphs are for trials where the critical stimulus was in the center of the display, and the bottom two graphs are for trials where the critical stimulus was away from the center of the display. For Observer 18, only an upper bound could be estimated for the smalleccentricity-fixation condition.

position of the rectangle is confounded with eccentricity. Thus, only the centered condition is relevant to the current arguments.

To check that this display artifact did not contribute to other tasks, the line-length experiment was replicated using the modified design of the rectangle-shape experiment. Observers 17 and 23 showed eccentricity reductions of $103 \%$ and $89 \%$ when the lines were centered and reductions of $95 \%$ and $95 \%$ when the lines were away from the center. Thus, lines at the center of the display were not perceived any differently than those away from the center of the display. We have confidence that this display artifact had little effect on the other experiments. ${ }^{6}$

\section{Discussion}

This experiment aimed to replicate the Experiment 1 for three new judgments: relative orientation, rectangle height, and rectangle shape. When properly controlled for all three judgments, eye movements nearly eliminated the 
effect of eccentricity. This experiment makes it plausible that the results found with Experiment 1 will generalize to a wide range of visual attributes. More conservatively, it seems plausible that the results will generalize to any single dimension of visual size and shape.

\section{GENERAL DISCUSSION}

In all of the experiments, performance improved when the observers moved their eyes to foveate individual stimuli. When appropriately measured, this improvement approached the performance possible with foveal viewing without eye movements for four of four tasks. Such an improvement is expected if memory for previous fixations survives the intervening eye movements. These results resembled the visual-direction-constancy results that showed no interference from eye movements and differed from visual-integration results that showed complete interference from eye movements.

One feature of these experiments was the use of unidimensional size and shape discriminations. While these tasks are common in visual psychophysics, they have only recently been used to study the details of visual memory (Palmer, 1988, 1989, 1990, 1991). The tasks were chosen because they yield a quantification of performance that is based on well-established theory in psychophysics. This quantification is what made possible the measurement of the degree of interference rather than a qualitative demonstration of some interference. Of course, these procedures must still be generalized to arbitrary visual patterns and linguistic stimuli.

\section{Issues}

Interference. The focus of this study is to measure the interference of multiple eye fixations on memory. Eye movements were manipulated by the instruction to fixate or move. An eye movement provides an opportunity to foveate a stimulus at the cost of possible interference from the eye movement. Experiment 1 demonstrated performance with movement instructions that matched foveal viewing, suggesting that moving one's eyes was all benefit and no cost. Experiments with three other tasks showed similar results. Thus, for size and shape attributes, there is no interfering effect of eye movement. This result goes beyond previous work in showing no interference rather than simply demonstrating some degree of surviving memory.

The question now is: Under what conditions will an eye movement interfere with memory? Interference was found with the visual-memory-integration paradigm of visualmemory research (e.g., Irwin, 1991; Irwin et al., 1983). Some interference was also found with the visualdirection-constancy paradigm when the stimulus was presented during the saccade. The methods of the current experiments can be adapted to measure the amount of interference in each of these other paradigms. Determining the amount of interference under different stimulus conditions will provide the data for developing a theory of the relevant memory systems.
Capacity. This study did not measure the perceptual capacity of a single eye fixation. In particular, performance was not measured as a function of the number of stimuli, as has been extensively studied elsewhere. For example, Palmer (1990) made measurements similar to those here and found that size and shape thresholds doubled when the number of stimuli was increased from one to four. Would a similar result be found if memory for a previous fixation was measured instead of memory for a brief presentation? No definite answer is possible yet, but the available evidence points to sharp capacity limits. One argument is that a comparison of Experiments 1 and 3 shows a similar capacity limit to that found in Palmer (1990). A second argument comes from the demonstrations of capacity-limit experiments reported by Irwin (1991) and by Lachter, Hayhoe, and Feldman (1991). We suspect that perceptual capacity of a single eye fixation is limited. If so, the reason for eye movements' causing little interference may be that relatively little information needs to be preserved across eye movements. The critical bottleneck may be in placing information into memory in the first place.

Code. What is the nature of the information remembered from a previous fixation? In particular, is it represented by a visual or an abstract code? To answer this question, we follow others in distinguishing between a memory and a store (e.g., Atkinson \& Shiffrin, 1968). A visual memory implies only that one can remember visual information. The memory's structure or codes may or may not be visual in any other sense. In contrast, a very short term visual store is a theoretical construct that has features analogous to visual stimuli. These features include a high information capacity and a sensitivity to visual manipulations such as masking and image translation (cf. Phillips, 1974). In the current situation, the experiments clearly measure visual memory because they use visual stimuli. But it is unlikely that they measure a very short term visual store. In particular, the paradigm requires that memories must survive an image translation caused by eye movements, and the results of Palmer (1990) demonstrate sharp capacity limits in a related task. Thus, we agree with Irwin (1991) that no very short term visual store survives an eye movement.

What does survive an eye movement? One possibility is a more durable visual store such as suggested by Phillips (1974) and Irwin (1991). By this hypothesis, there is a limited-capacity store that codes visual information in a schematic form. This information is not degraded by masking or image translation. It differs from verbal or more abstract codes in directly representing spatial information. In the current experiments, this hypothesis is supported by the invariance of results with changes in test uncertainty. No abstract categorization strategy was essential to avoiding interference from eye movements.

\section{The Fixation-Sampling Hypothesis}

This article began by describing the fixation-sampling hypothesis: The inputs to perceptual processes are samples of a scene obtained from individual eye fixations. 
Such a hypothesis requires memory for information from previous fixations. The current results establish several instances where visual information is remembered without interference. This adds to a list of such cases that probably includes visual direction and reading. One immediate problem is to establish cases besides visual-memory integration where information does not survive. A more general issue is whether eye fixations are always the unit of sampling. This is particularly in doubt for moving stimuli and pursuit eye movements. Such situations may well yield interesting alternatives to the fixation-sampling hypothesis.

\section{Conclusions}

The goal of this study was to measure how much multiple eye fixations interfere with visual memory. Several experiments showed that size and shape attributes were remembered from previous fixations with little or no interference. This result was replicated with four attributes and several procedural variations. It is compatible with the hypothesis that no very short term visual store survives an eye movement. Instead, information must be recoded into some kind of limited-capacity memory to survive from eye fixation to eye fixation.

\section{REFERENCES}

Atkinson, R. C., Shiffrin, R. M. (1968). Human memory: A proposed system and its control processes. In K. W. Spence \& J. T. Spence (Eds.), The psychology of learning and motivation (Vol. 2, pp. 89195). New York: Academic Press.

Balota, D. A., Pollatsex, A., \&ayner, K. (1985). The interaction of contextual constraints and parafoveal visual information in reading. Cognitive Psychology, 17, 364-390.

CowEy, A., * RoLLs, E. T. (1974). Human cortical magnification factor and its relation to visual acuity. Experimental Brain Research, 21, 447-454.

Di LoLLo, V. (1977). Temporal characteristics of iconic memory. Nature, 267, 241-243.

FARrell, J. E., \& Desmarais, M. (1990). Equating characteridentification performance across the visual field. Journal of the $O p$ tical Society of America, A7, 152-159.

Hansen, R. M., Ska venski, A. A. (1985). Accuracy of spatial localizations near the time of saccadic eye movements. Vision Research, 25, 1077-1082.

Hayhoe, M., Lachter, J., \& Feldman, J. (1991). Integration of form across saccadic eye movements. Perception, 20, 393-402.

Henderson, J. M., Pollatsek, A., Rayner, K. (1987). Effects of foveal priming and extrafoveal preview on object identification. Journal of Experimental Psychology: Human Perception \& Performance, 13, 449-463.

IRWIN, D. E. (1991). Information integration across saccadic eye movements. Cognitive Psychology, 23, 420-456.

IrWIN, D. E., \& BrowN, J. S. (1988, November). Visual integration across saccadic eye movements. Paper presented at the meeting of the Psychonomic Society, Chicago.

IRwIN, D. E., YANTIS, S., \& JoNIDES, J. (1983). Evidence against visual integration across saccadic eye movements. Perception \& Psychophysics, 34, 49-57.

IRWIN, D. E., ZACKS, J. L., \& Brown, J. S. (1990). Visual memory and the perception of a stable visual environment. Perception \& Psychophysics, 47, 35-46.

KLEIN, S. A., LEVI, D. M. (1987). The position sense of the peripheral retina. Joumal of the Optical Society of America, A4, 1544-1553.
Kowler, E., Steinman, R. M. (1977). The role of small saccades in counting. Vision Research, 17, 141-146.

Kowler, E., Steinman, R. M. (1979). Miniature saccades: Eye movements that do not count. Vision Research, 19, 105-108.

Lachter, J., Hayhoe, M., Feldman, J. (1991, May). Capacity limitations in the integration of information across saccades. Paper presented at the meeting of the Association for Research in Vision and Ophthalmology, Sarasota, FL.

MATIN, L. (1986). Visual localization in eye movements. In K. R. Boff, L. Kaufman, \& J. P. Thomas (Eds.), Handbook of perception and human performance (Vol. l, pp. 20-1-20-45). New York: Wiley.

Matin, L., Matin, E., PearCe, D. G. (1969). Visual perception of direction when voluntary saccades occur: $I$. Relation of visual direction of a fixation target extinguished before a saccade to a flash presented during the saccade. Perception \& Psychophysics, 5, 65-80.

Matin, L., Matin, E., * Pola, J. (1970). Visual perception of direction when voluntary saccades occur: II. Relation of visual direction of a fixation target extinguished before a saccade to a subsequent test flash presented before the saccade. Perception \& Psychophysics, 8 , 9-14.

McConkIe, G. W., RAYNer, K. (1975). The span of the effective stimulus during a fixation in reading. Perception \& Psychophysics, 17, 578-586.

McCoNkIE, G. W., \& ZoLA, D. (1979). Is visual information integrated across successive fixations in reading? Perception \& Psychophysics, 25, 221-224.

McConkie, G. W., Zola, D., Blanchard, H.E., Wolverton, G. S. (1982). Perceiving words during reading: Lack of facilitation from prior peripheral exposure. Perception \& Psychophysics, 32, 271-281.

O'RegAN, J. K. (1984). Retinal versus extraretinal influences in flash localization during saccadic eye movements in the presence of a visible background. Perception \& Psychophysics, 36, 1-14.

O'RegaN, J. K., \& LÉvy-SChoEN, A. (1983). Integrating visual information from successive fixations: Does trans-saccadic fusion exist? Vision Research, 23, 765-768.

PALMER, J. (1988). Very short-term visual memory for size and shape. Perception \& Psychophysics, 43, 278-286.

PAlmer, J. (1989). A multiplicative attenuation model of attention. Paper presented at the meeting of the Society of Mathematical Psychology, Irvine, $\mathbf{C A}$.

PAlmer, J. (1990). Attentional limits on the perception and memory of visual information. Joumal of Experimental Psychology: Human Perception \& Performance, 16, 332-350.

PALMER, J. (1991). Isolating the components of very-short-term visual memory. Bulletin of the Psychonomic Society, 29, 399-402.

Palmer, J., Ames, C., Lindsey, D. (in press). Measuring the effect of attention on simple visual search. Journal of Experimental Psychology: Human Perception \& Performance.

PhILliPs, W. A. (1974). On the distinction between sensory storage and short-term visual memory. Perception \& Psychophysics, 16, 283-290.

Pollatsek, A., Rayner, K., \& Coluns, W. E. (1984). Integrating pictorial information across eye movements. Journal of Experimental Psychology: General, 113, 426-442.

RAYNER, K. (1975). The perceptual span and peripheral cues in reading. Cognitive Psychology, 7, 65-81.

RAYNER, K., Fisher, D. L. (1987). Letter processing during eye fixations in visual search. Perception \& Psychophysics, 42, 87-100.

RAYNER, K., McConkIE, G. W., \& Zola, D. (1980). Integrating information across eye movements. Cognitive Psychology, 12, 206-226.

Rayner, K., Pollatsek, A. (1983). Is visual information integrated across saccades? Perception \& Psychophysics, 34, 39-48.

SChungensiepen, K. H., CAmpBell, F. W., Legge, G. E., W Walker, T. D. (1986). The importance of eye movements in the analysis of simple patterns. Vision Research, 26, 1111-1117.

Sun, J. S., \& IRWIN, D. E. (1987). Retinal masking during pursuit eye movements: Implications for spatiotopic visual persistence. Journal of Experimental Psychology: Human Perception \& Performance, 13, 140-145. 


\section{NOTES}

1. For individual observers, the standard errors of the mean are based on replications over the sessions of the experiment. This by-session analysis results in slightly different threshold estimates than those graphed in the figures. In the figures and for the analysis across subjects, the threshold estimates were based on probit analysis of the pooled data across all blocks. These pooled analyses are usually preferred because they show less bias than the by-session analyses. However, analysis of individual sessions was necessary to obtain conservative estimates of the standard errors of the mean percentage reduction of the eccentricity effect.

2. For individual observers, the standard errors of the mean are based on replications over the sessions of the experiment.

3. No meaningful standard error of the mean could be calculated because of idiosyncratic data for some of the individual sessions. Instead, we report the statistic for the pooled data alone.

4. Because of perfect performance in the small-eccentricity-fixation condition, $75 \%$ is only a lower bound on the reduction of the eccentricity effects.

5. Standard errors of the mean could not be calculated in these conditions because near-perfect overall performance resulted in some blocks with no errors.
6. We speculate that the differences observed with the rectangle-shape tasks were due to an artifact of displaying rectangles away from the center of the display. Four possible sources of error cannot be distinguished here: (1) a physical distortion at the edge of the display, (2) a perceptual distortion near the edge of the screen due to the nearby visual border, (3) an error due to the display being presented on a screen that curves away from the observer rather than remaining in the parallel frontal plane, and (4) a perceptual error due to imperfect size constancy across the parallel frontal plane. Physical measurements of the display found no measurable error, but this physical measurement was no more accurate than $0.5 \mathrm{~mm}$, which is $3 \%$ of the height of the standard rectangle. The systematic distortion due to the curved screen was $1 \%$, and the difference in visual angle from the parallel frontal plane to a perimeter display was $1 \%$. Although all of these errors were small, they may contribute to the threshold in the rectangle task because the thresholds themselves were as small as $1 \%$ of the rectangles' height.

(Manuscript received July 12, 1991; revison accepted for publication March 17, 1992.) 